\title{
How Much of Root Cause Analysis Translates into Improved Patient Safety: A Systematic Review
}

\author{
Jimmy Martin-Delgado a Alba Martínez-García ${ }^{b}$ Jesús María Aranaz ${ }^{c}$ \\ José L. Valencia-Martín ${ }^{c}$ José Joaquín Mira ${ }^{a, b, d}$ \\ ${ }^{a}$ Atenea Investigation Group, Fundación para el Fomento de la Investigación Sanitario y Biomédica de la \\ Comunidad Valenciana (FISABIO), Valencia, Spain; ${ }^{b}$ Department of Health Psychology, Universidad \\ Miguel Hernández, Alicante, Spain; ' Preventive Medicine Service, Hospital Ramon y Cajal, Madrid, Spain; \\ ${ }^{d}$ Alicante-Sant Joan d'Alacant Health District, Alicante, Spain
}

\section{Significance of the Study}

- Root cause analysis is a widespread technique used in the last two decades to investigate latent causes of adverse events.

- Lack of expertise and time and a weak legal framework are some of the difficulties in fulfilling action plans from root cause analysis.

- It is not clear if root cause analysis is effective in preventing the recurrence of adverse events.

\section{Keywords}

Root cause analysis · Patient safety · Quality assurance ·

Translational medicine

\begin{abstract}
Objectives: The aim of this systematic review was to consolidate studies to determine whether root cause analysis ( $R C A$ ) is an adequate method to decrease recurrence of avoidable adverse events (AAEs). Methods: A systematic search of databases from creation until December 2018 was performed using PubMed, Scopus and EMBASE. We included articles published in scientific journals describing the practical usefulness in and impact of RCA on the reduction of AAEs and whether professionals consider it feasible. The Mixed Methods Appraisal Tool was used to assess the quality of studies. Results: Twenty-one articles met the inclusion crite-
\end{abstract}

ria. Samples included in these studies ranged from 20 to 1,707 analyses of RCAs, AAEs, recommendations, audits or interviews with professionals. The most common setting was hospitals ( $86 \% ; n=18$ ), and the type of incident most analysed was AAEs, in $71 \%(n=15)$ of the cases; $47 \%(n=10)$ of the studies stated that the main weakness of RCA is its recommendations. The most common causes involved in the occurrence of AEs were communication problems among professionals, human error and faults in the organisation of the health care process. Despite the widespread implementation of RCA in the past decades, only 2 studies could to some extent establish an improvement in patient safety due to RCAs. Conclusions: RCA is a useful tool for the identification of the remote and immediate causes of safety incidents, but not for implementing effective measures to prevent their recurrence.

(C) 2020 The Author(s)

Published by S. Karger AG, Basel
(C) 2020 The Author(s)

Published by S. Karger AG, Basel

This is an Open Access article licensed under the Creative Commons Attribution-NonCommercial-4.0 International License (CC BY-NC) (http://www.karger.com/Services/OpenAccessLicense), applicable to the online version of the article only. Usage and distribution for commercial purposes requires written permission.
Jimmy Martin-Delgado

Foundation for the Promotion of Health and Biomedical Research

Hospital Universitario de Sant Joan d'Alacant

Carretera Nacional 332, ES-03550 Alicante (Spain)

jimmy.martind@umh.es 


\section{Introduction}

Root cause analysis (RCA) emerged in the health care field almost 20 years ago. This technique is used worldwide to understand the remote and direct factors favouring the occurrence of an avoidable adverse event (AAE) [1], and improvement of patient safety [2].

Three studies have analysed the utility and limitations of this technique [3-5], all agreeing that barriers to implementing RCA correctly remain, and most of these were focused on active errors and not latent causes. The published results invite us to reflect on whether the impact of this technique in practice is proving to be all that is theoretically expected, first, because substantial resources and human talent are required [5], and, second, because many of the recommendations made in the framework of this technique are not applied failing to prevent the same incidents from recurring [6].

It is one thing to perform a multidisciplinary and multicausal analysis of why an incident occurred, and another, totally different thing to ensure that the proposals derived from this analysis to avoid the recurrence of safety incidents are implemented successfully. The usefulness of RCA may depend on the safety culture of health institutions, decision-making procedures and middle management leadership styles. The objective of this study was to assess the usefulness (i.e., its capacity to improve patient safety) of RCA in health care practice in order to avoid recurrence of AAEs.

\section{Methods}

We conducted a systematic review of studies published in scientific journals from database creation until December 2018 on the practical usefulness of RCA in the reduction of recurrent AAEs.

\section{Eligibility Criteria}

Only studies that analysed the efficacy of RCAs in the improvement of patient safety and studies that analysed the views of professionals on its feasibility were included. This study was designed to address whether the results of RCA improve patient safety, whether the RCA methodology has been fully carried out, and whether health professionals consider it feasible.

The MOOSE protocol was followed. Studies published in scientific journals were included to describe the AEs commonly analysed and the practical usefulness of RCA in the reduction of AAEs or its limitations in improving patient safety. No exclusions were made based on the year of publication, the design of the study, or language. Both quantitative and qualitative studies were included. Research taking a descriptive approach was also included.
Search Strategy

The review of published studies was carried out on MEDLINE, Scopus and EMBASE. The search strategies were defined based on the combination of keywords related to RCA (("root cause analysis"[MeSH Terms] OR ("root"[All Fields] AND "cause"[All Fields] AND "analysis"[All Fields]) OR "root cause analysis"[All Fields]) AND ("delivery of health care"[MeSH Terms] OR ("delivery"[All Fields] AND "health"[All Fields] AND "care"[All Fields]) OR "delivery of health care"[All Fields])) AND ("patient safety" [MeSH Terms] OR ("patient"[All Fields] AND "safety"[All Fields]) OR "patient safety"[All Fields]), and using the Boolean operators "AND" and "OR."

In a complementary manner, we conducted manual searches in Google Scholar for the identification of grey literature using the same search equation. We also carried out a manual search based on the bibliographic references of the selected publications.

\section{Data Extraction}

J.M.-D. and A.M.-G. independently reviewed all potentially relevant studies. When no consensus was achieved between them, a third researcher (J.J.M.) reviewed the study to reach a consensus. All reviewers were capable of understanding Spanish, English and French, and studies were included in local languages if they included an English abstract, which allowed the authors to decide whether they had to be included for full text reading. No unpublished studies were found, nor was there a need to contact any of the authors of the studies included. Data extracted from each study included country, review committee, inclusion of managers, inclusion of personnel involved, design, database used, employee review method, sample (RCA number, recommendations, audits or interviews conducted), the type of event analysed and the main findings. In addition, where possible, information was extracted on the applicability of the RCAs and the interest of health professionals in the RCA.

Data Synthesis

For each of the above variables, categories of possible options were generated and the presence or absence of information in each variable was coded. Heterogeneity in the methods and measures applied was described when possible. Numbers of participants and AEs were also included. AAEs were classified as sentinel where applicable.

\section{Quality Evaluation}

For the assessment of quality of the included studies, J.M.-D. and A.M.-G. used the Mixed Methods Appraisal Tool (MMAT) [7]. The results led to an overall score for methodological quality, varying from $40-60 \%$ (moderate quality) and $80 \%$ (considerable quality) to $100 \%$ (high quality). None of the studies were excluded based on the quality assessment.

\section{Results}

A total of 169 studies were identified, of which 127 remained after exclusion of duplicates. Seventy-seven were excluded as these studies did not include results concerning the effectiveness of RCA in decreasing the number of 
Fig. 1. Study selection flow diagram. RCA, root cause analysis.

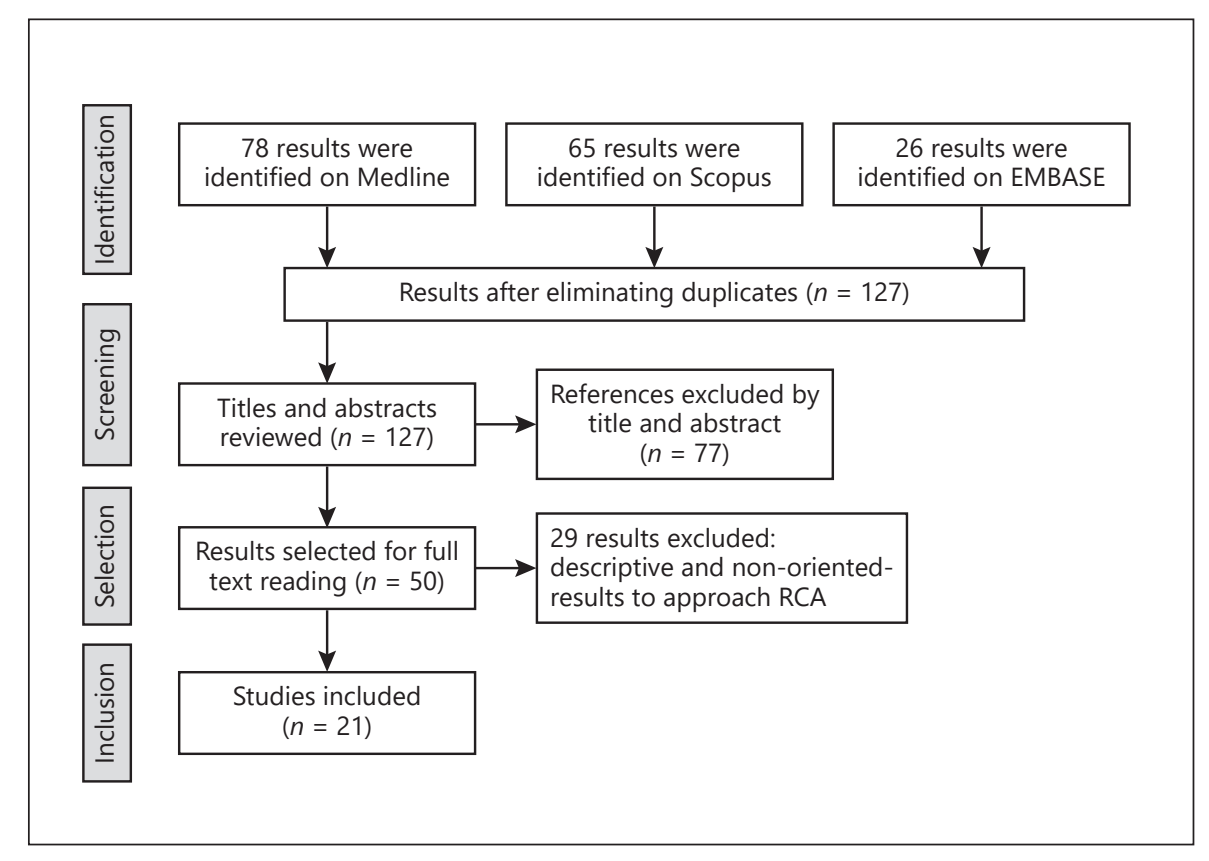

AAEs. Finally, 21 papers meeting all inclusion criteria were included (Fig. 1).

The samples of the studies show a high variability, from 20 to 1,707 subjects with analysed data (RCA number, recommendations, audits or interviews conducted). The studies were mainly conducted in North America (52\%; $n=11)$, Australia (19\%; $n=4)$ and Europe (14\%; $n=3$ ); $57 \%$ were quantitative studies (6 longitudinal and 6 cross-sectional), 1 study was a randomised controlled trial, and the rest were qualitative analyses. The most common setting was hospitals ( $86 \% ; n=18)$. The type of incident most analysed was AAEs, in $71 \%(n=15)$ of the studies, where sentinel events represented $13 \%(n=2)$ of the AEs, and serious AEs classified as Severity Assessment Code 1 (for Queensland Health) or 3 (for the Department of Veterans Affairs) accounted for $20 \%(n=3)$, while for the remaining 10 studies, information about severity was not disclosed. Incidents related to safety made up 5\% $(n=1)$, and in the rest of the included studies, interviews or mock-ups were implemented.

In only 5 studies (24\%) were managers or coordinators included, and in only 1 study were personnel from the service taken into consideration. Independent hospital databases and national or regional databases were mostly used, each accounting for $29 \%$ of the studies. Although the Veterans Affairs National Center for Patient Safety database was used in 5 of the studies (24\%), they also examined individuals. For the rest of the cases, databases were not used.

\section{Quality Evaluation}

Of the 21 articles, 9 were rated as having a moderate quality (MMAT 40-60\%), 5 were rated as having a considerable quality (MMAT $80 \%$ ), and 7 were rated as high quality (MMAT 100\%). The key features of each study are presented in Table 1 [3-6, 8-24].

\section{Utility of RCA}

Only in 2 studies (9\%) could it be established that RCAs contributed to the improvement of patient care to some extent. In these studies, only 54 RCAs were reviewed. In 3 other studies (15\%), the authors did not conclude that their implementation contributed to the improvement of patient safety, and in 10 (50\%) of them, the recommendations made were weak, which did not lead to a reduction of AEs.

Some studies warned that sometimes poorly designed action plans and measures may generate new risks and may be insufficient to prevent the occurrence of new events $[5,6,10,16]$. In the study carried out by Hibbert et al. [4], in which the effectiveness and sustainability of the proposals arising from 227 RCAs were analysed, it was found that $72 \%$ of the recommendations categorised as relevant were not formulated and that the most common recommendations did not refer to latent causes in clinical practices but to active errors. Likewise, other studies $[3,6]$ stated that most of the proposed recommendations focused on active errors and neglected latent causes, which provides short-term solutions but only par- 


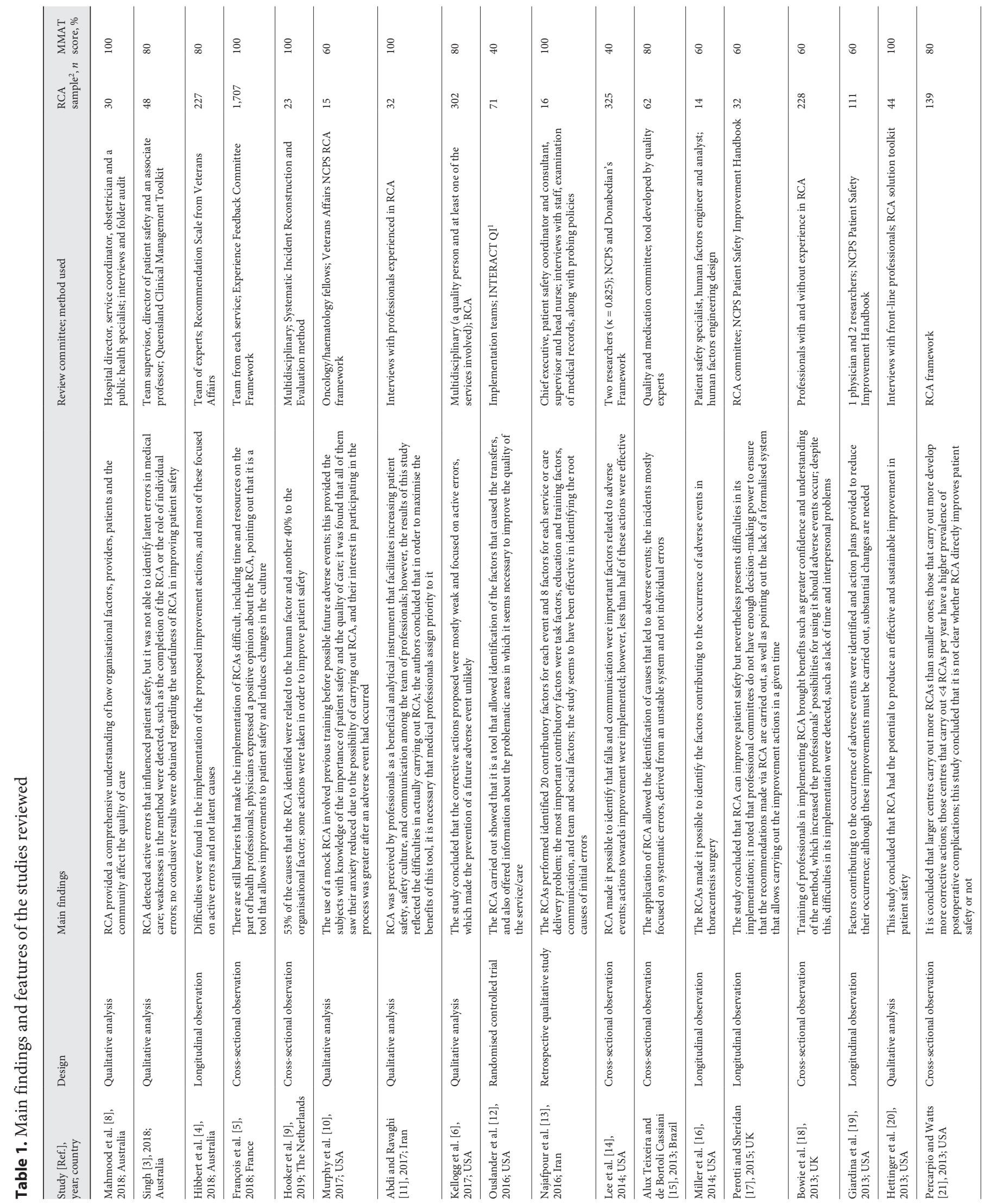




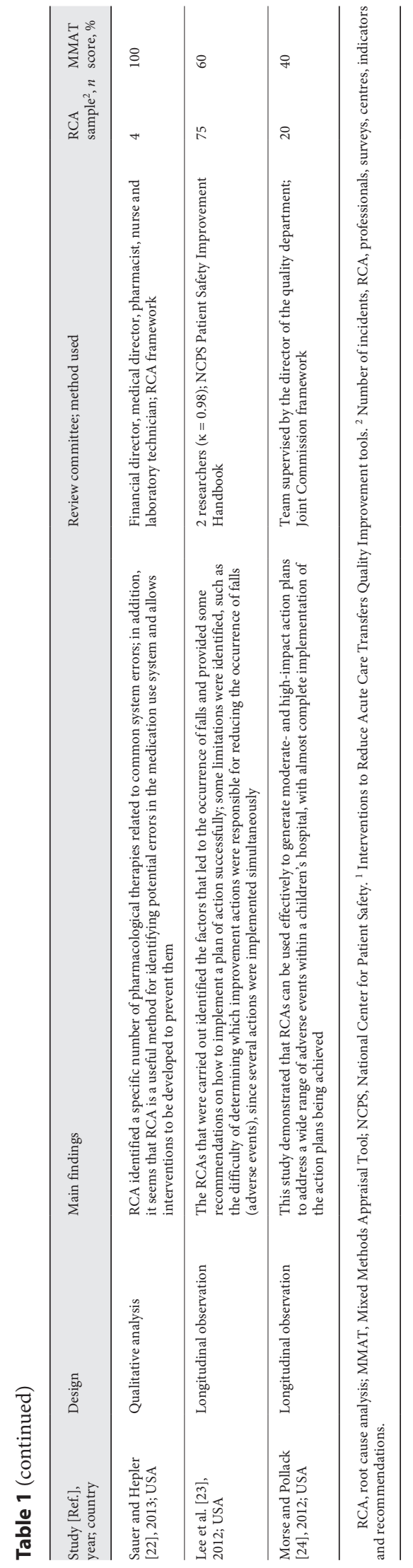

tially helps to avoid future incidents. However, 1 study (5\%) did demonstrate the usefulness of RCA and its recommendations [20].

Three studies (15\%) showed that the RCA method was not applied properly [3, 5, 25]. François et al. [5] stated that only $23 \%$ of the 98 events selected for in-depth analysis covered all the domains defined by the method. Singh [3] pointed out that $65 \%$ of the RCAs carried out were not properly completed. In 5 studies (25\%), the involvement of the professionals in the realisation of the RCA was analysed. While these appear to suggest a consensus that the relevance of RCA to establishing improved procedures caused it to be perceived as a beneficial analytical instrument, the researchers concluded that professionals encounter difficulties in conducting RCAs $[8,14]$.

\section{Discussion}

The literature collected analysed the usefulness of RCA as a process that allows the determination of factors that contribute to the occurrence of AAEs. Most of these were caused by communication problems among professionals [3, 13, 14, 19], human error [9] and, finally, faults in the organisation of the health care process $[3,22]$.

\section{Weaknesses in Using RCA}

Despite the fact that for professionals, RCA is a tool that allows improvements to the safety culture through the prevention of future AAEs $[3,5]$, studies have identified the main weaknesses diminishing the usefulness of RCA in the framework of patient safety [5]. RCA is an extensive process that requires proper qualifications and attitudes. Studies have shown that the RCA method is often not properly applied, which directly affects the rigor and depth of the analysis of this tool $[3,5,25]$. The most common difficulties are a lack of time and resources of the work teams. Although none of the studies reviewed refers to how much time is needed to perform an RCA, in the study conducted by McGraw and Drennan [26], the results of an RCA investigation of pressure ulcers showed that a nurse can take up to $20 \mathrm{~h}$ to complete it. These factors directly affect the RCA process, hindering its complete execution [5].

Lack of time does not seem to be the only element that hinders the performance of RCAs; describing the events from a perspective different from individual error is challenging to professionals, who are sometimes reluctant to participate because of the distrust generated by possible future consequences. The belief that there is a culture fo- 
cused on searching "those responsible" - in addition to creating tension in the work environment that may cause interprofessional problems $[5,18]$ - is one of the main reasons for professionals refusing to participate in incident reporting systems [27].

\section{Impact on Reducing AAEs}

None of the studies reviewed assessed the ability of this process to reduce future AAEs. Moreover, in the study by Lee et al. [23], difficulties were found in determining whether the improvement actions implemented after the RCA were able to reduce the occurrence of AAEs, as the action plans did not follow a pattern of controlled implantation. On the other hand, Morse and Pollack [24] and Sauer and Hepler [22] were the only ones that found sufficient follow-up time, which allowed the determination of whether the recommendations agreed on in the RCA reduced the occurrence of AAEs to some degree. In order to increase the commitment and interest of health professionals in carrying out RCA, some studies have evaluated the usefulness of a mock RCA as prior training to provide medical staff with basic knowledge about patient safety and the methodology of this tool. The results showed that its use raised their confidence in being able to perform RCA and decreased their anxiety in the face of a possible AE $[10,18]$. This is supported by Boussat et al. [28], who found that professionals who were involved in Experience Feedback Committees had better scores on the Hospital Survey on Patient Safety Culture, especially in non-punitive response to error, communication and organisational learning dimensions.

\section{Improvements in Introducing Changes}

The studies reviewed show that managers and the personnel involved in AAEs had a low participation in the committees in charge of carrying out RCA. Peerally et al. [25] pointed out the need to professionalise the investigation of incidents by involving experts, as well as patients and family members, who can provide a unique perspective on the care received. Including those professionals who have been directly involved in the event also seems to have positive consequences, as it can contribute to the recovery of second victims by making them part of the solution [29]. Despite the progress and improvement in quality of care, the involvement of report systems such as RCA, especially the implementation of measures adopted, does not seem to be successful. Most of the included countries have so-called apology laws (39 states of the USA have Medical Professional Apologies Statutes; e.g., the Maryland Courts and Judicial Proceedings Code Ann.
$\$ 10-92041$ ), which aim to improve the relationship with the patient and their families, allowing them to provide information that improves the quality of care [27]. However, the presence of the professionals involved in an $\mathrm{AE}$ in RCA committees was mentioned in only 1 study [6], and in none of the cases were relatives present.

Although AEs are sometimes inevitable and part of the uncertainty of medical practice, the ultimate goal of the RCA is to help to produce solutions and apply necessary measures to ensure they do not happen again, that there is a lower probability of their occurrence, or that if they occur the possible consequences are mitigated. Although some studies have demonstrated the usefulness of RCA and its recommendations [24], most published studies found that just over half of the recommendations that resulted from RCA were not useful enough to prevent the same incidents from recurring in the future $[6,14,25]$. For this reason, researchers have proposed RCA-based tools that seek greater involvement of frontline professionals in the implementation of proposals to prevent the recurrence of serious AEs [30]. The non-existence of a formalised system that allows these recommendations to be addressed in a specific period of time, as well as the limited power of RCA committees to ensure compliance with these improvement actions [17], hinders implementing the proposed actions. It must be borne in mind that, normally, the people who will have to assume the responsibility of approving the measures suggested by the RCAs and the professionals who must implement these measures are usually not those who participated directly in the RCA. This makes it difficult for the proposals to be carried out.

It seems, therefore, that RCA is a process with considerable validity through which much may be learned about incidents $[12,15]$. However, it does not seem to produce enough benefits to address and resolve the problem [23, 25 ] and, thus, to avoid possible AAEs. As explained by Najafpour et al. [13], RCA is a reactive method for investigating an event and finding its underlying factors. This method could provide answers to questions related to what happened, and how and why it happened, and should be used to design preventive interventions. It should be performed using an outlined framework process of investigation and analysis of clinical incidents performed by professionals with experience in patient safety and should include at least one manager and one of the personnel involved in it.

\section{Limitations}

Some of the limitations encountered are that most of the published data related to RCAs pertain to analysing 
the method of determining whether RCA is being implemented. Only reported results and quotations were assessed. Finally, little of the scarce evidence available regarding the impact - which was our objective - of carrying out RCAs can actually contribute to reducing the recurrence of the same incident, which may be due to the limited decision-making power of committees or to the proposed plan of action not being followed up in time.

\section{Conclusions}

Although early studies suggested that RCAs are effective in promoting ideas for preventing recurrence, more recent studies do not confirm these findings. A common gap that limits the effectiveness of this tool lies in the fact that RCAs are not usually accompanied by subsequent control of whether the devised improvement plan is carried out. Further research should focus on how to translate the results of ACR recommendations into practice to prevent recurrence of AAEs.

\section{Statement of Ethics}

For this systematic review, the MOOSE protocol was followed.

\section{Conflict of Interest Statement}

The authors have no conflicts of interest to declare.

\section{Funding Sources}

This research was funded by the PROMETEO Research Program, Consellería de Educación, Investigación, Cultura y Deporte, Generalitat Valenciana (Prometeu173).

\section{Author Contributions}

The conception and design were made by J.J.M.; the data were collected by A.M.-G. and J.M.-D.; the article was supervised by J.J.M.; all the authors contributed to writing, revising and approving the final draft.

\section{References}

1 Ruiz-Lopez P, Aranaz J. Analisis Causa Raiz. Root Cause Analysis. La gestión sanitaria orientada hacia la calidad y seguridad de los pacientes. 2nd ed. Madrid: Fundación MAPFRE; 2017. pp. 563-6.

2 Australian Commission on Safety and Quality in Health Care. National Safety and Quality Health Service Standards (September 2012). 2012

3 Singh K. Lifting the lid on root cause analysis: a document analysis. Saf Sci. 2018;107:10918.

4 Hibbert PD, Thomas MJ, Deakin A, Runciman WB, Braithwaite J, Lomax S, et al. Are root cause analyses recommendations effective and sustainable? An observational study. Int J Qual Health Care. 2018 Mar;30(2):12431.

5 François P, Lecoanet A, Caporossi A, Dols AM, Seigneurin A, Boussat B. Experience feedback committees: a way of implementing a root cause analysis practice in hospital medical departments. PLoS One. 2018 Jul; 13(7):e0201067.

6 Kellogg KM, Hettinger Z, Shah M, Wears RL, Sellers CR, Squires M, et al. Our current approach to root cause analysis: is it contributing to our failure to improve patient safety? BMJ Qual Saf. 2017 May;26(5):381-7.

7 Hong QN, Fàbregues S, Bartlett G, Boardman F, Cargo M, Dagenais P, et al. The Mixed Methods Appraisal Tool (MMAT) version 2018 for information professionals and researchers. Educ Inf. 2018 Dec;34(4):285-91.
8 Mahmood MA, Mufidah I, Scroggs S, Siddiqui AR, Raheel $\mathrm{H}$, Wibdarminto $\mathrm{K}$, et al. Root-Cause Analysis of Persistently High Maternal Mortality in a Rural District of Indonesia: Role of Clinical Care Quality and Health Services Organizational Factors. BioMed Res Int. 2018 Feb;2018:3673265.

9 Hooker AB, Etman A, Westra M, Van der Kam WJ. Aggregate analysis of sentinel events as a strategic tool in safety management can contribute to the improvement of healthcare safety. Int J Qual Heal Care. 2019 Mar;31(2): 110-6.

10 Murphy M, Duff J, Whitney J, Canales B, Markham MJ, Close J. Implementation of a mock root cause analysis to provide simulated patient safety training. BMJ Open Qual. 2017 Oct;6(2):e000096.

11 Abdi Z, Ravaghi H. Implementing root cause analysis in Iranian hospitals: challenges and benefits. Int J Health Plann Manage. 2017 Apr;32(2):147-62.

12 Ouslander JG, Naharci I, Engstrom G, Shutes J, Wolf DG, Alpert G, et al. Root Cause Analyses of Transfers of Skilled Nursing Facility Patients to Acute Hospitals: Lessons Learned for Reducing Unnecessary Hospitalizations. J Am Med Dir Assoc. 2016 Mar;17(3):256-62.

13 Najafpour Z, Jafary M, Saeedi M, Jeddian A, Adibi $\mathrm{H}$. Effect size of contributory factors on adverse events: an analysis of RCA series in a teaching hospital. J Diabetes Metab Disord. 2016 Jul;15(1):27.
14 Lee A, Mills PD, Neily J, Hemphill RR. Root cause analysis of serious adverse events among older patients in the Veterans Health Administration. Jt Comm J Qual Patient Saf. 2014 Jun;40(6):253-62.

15 Alux Teixeira TC, de Bortoli Cassiani SH Root cause analysis of falling accidents and medication errors in hospital. Acta Paul Enferm. 2013;26(4):100-7.

16 Miller KE, Mims M, Paull DE, Williams L, Neily J, Mills PD, et al. Wrong-side thoracentesis: lessons learned from root cause analysis. JAMA Surg. 2014 Aug;149(8):774-9.

17 Perotti V, Sheridan MM. Root cause analysis of critical events in neurosurgery, New South Wales. ANZ J Surg. 2015 Sep;85(9):626-30.

18 Bowie P, Skinner J, de Wet C. Training health care professionals in root cause analysis: a cross-sectional study of post-training experiences, benefits and attitudes. BMC Health Serv Res. 2013 Feb;13(1):50.

19 Giardina TD, King BJ, Ignaczak AP, Paull DE, Hoeksema L, Mills PD, et al. Root Cause Analysis Reports Help Identify Common Factors in Delayed Diagnosis and Treatment of Outpatients. Health Aff (Millwood). 2013 Aug;32(8):1368-75.

20 Hettinger AZ, Fairbanks RJ, Hegde S, Rackoff AS, Wreathall J, Lewis VL, et al. An evidencebased toolkit for the development of effective and sustainable root cause analysis system safety solutions. J Healthc Risk Manag. 2013, 33(2):11-20. 
21 Percarpio KB, Watts BV. A cross-sectional study on the relationship between utilization of root cause analysis and patient safety at 139 Department of Veterans Affairs medical centers. Jt Comm J Qual Patient Saf. 2013 Jan; 39(1):32-7.

22 Sauer BC, Hepler CD. Application of systemlevel root cause analysis for drug quality and safety problems: a case study. Res Social Adm Pharm. 2013 Jan-Feb;9(1):49-59.

23 Lee A, Mills PD, Watts BV. Using root cause analysis to reduce falls with injury in the psychiatric unit. Gen Hosp Psychiatry. 2012 May-Jun;34(3):304-11.

24 Morse RB, Pollack MM. Root cause analyses performed in a children's hospital: events, action plan strength, and implementation rates. J Healthc Qual. 2012;34(1):55-61.
25 Peerally MF, Carr S, Waring J, Dixon-Woods M. The problem with root cause analysis. BMJ Qual Saf. 2017 May;26(5):417-22.

26 McGraw C, Drennan VM. Evaluation of the suitability of root cause analysis frameworks for the investigation of community-acquired pressure ulcers: a systematic review and documentary analysis. J Clin Nurs. 2015 Feb;24(34):536-45.

27 Mira Solves JJ, Romeo Casabona CM, Urruela Mora A, Agra Varela Y, Astier Peña P, Lorenzo Martinez S, et al. La seguridad jurídica de los profesionales sanitarios. Un requisito para lograr una mayor calidad asistencial. Derecho y Salud. 2017;27(2):94-110.
28 Boussat B, Seigneurin A, Giai J, Kamalanavin $\mathrm{K}$, Labarère J, François $\mathrm{P}$. Involvement in Root Cause Analysis and Patient Safety Culture among Hospital Care Providers. J Patient Saf. 2017, Online ahead of print.

29 Mira Solves JJ, Carrillo I, Guilabert M, Valencia-Martín JL, Aranaz Andrés JM, Martin J. Root Cause? Yes of course ... but then what? Rev Panam Salud Publica. 2019 Jun;43:e51.

30 Carrillo I, Mira JJ, Vicente MA, Fernandez C, Guilabert M, Ferrús L, et al. Design and Testing of BACRA, a Web-Based Tool for Middle Managers at Health Care Facilities to Lead the Search for Solutions to Patient Safety Incidents. J Med Internet Res. 2016 Sep;18(9): e257. 